\title{
Maximum Frustration in Bipartite Signed Graphs
}

\author{
Garry Bowlin \\ gsbowlin@gmail.com
}

\begin{abstract}
Submitted: April 3, 2012; Accepted: Oct 11, 2012; Published: Oct 25, 2012
Mathematics Subject Classification: 05C22, 91D30
\end{abstract}

\begin{abstract}
A signed graph is a graph where each edge is labeled as either positive or negative. A circle is positive if the product of edge labels is positive. The frustration index is the least number of edges that need to be removed so that every remaining circle is positive. The maximum frustration of a graph is the maximum frustration index over all possible sign labellings. We prove two results about the maximum frustration of a complete bipartite graph $K_{l, r}$, with $l$ left vertices and $r$ right vertices. First, it is bounded above by

$$
\frac{\operatorname{lr}}{2}\left(1-\frac{1}{2^{l-1}}\left(\begin{array}{l}
l-1 \\
\left\lfloor\frac{l-1}{2}\right\rfloor
\end{array}\right)\right) .
$$

Second, there is a unique family of signed $K_{l, r}$ that reach this bound. Using this fact, exact formulas for the maximum frustration of $K_{l, r}$ are found for $l \leqslant 7$.
\end{abstract}

\section{Introduction}

In 1954, Harary published "On the notion of balance of a signed graph", introducing the notion of a signed graph [6]. In his paper, Harary defined a signed graph as a graph whose edge set has been partitioned into positive and negative edges. He called a circle positive if it had an even number of negative edges, and he called a signed graph balanced if every circle was positive. He then gave both necessary and sufficient conditions for balance.

Four years after Harary's paper, Abelson and Rosenberg, [1], proposed a measure of imbalance, called frustration index (which they called "complexity" and which Harary called the "line index of balance"). They also gave an upper bound on the frustration index for simple graphs on $n$ vertices, which Petersdorf proved in [8]. In addition, Petersdorf proved that for each $n$, the signed graph that obtain this bound is unique (up to switching).

In [10], Solé and Zaslavsky discovered that the covering radius of the cutset code of a graph is equal its maximum frustration index. In particular, they showed that the 
covering radius of the Gale-Berlekamp codes were equal to the maximum frustration index of complete bipartite graphs.

In [5], Graham and Sloane show that the covering radius of the $l \times r$ rectangular Gale-Berlekamp code is bounded above by

$$
\operatorname{lr}\left(\frac{1}{2}-\frac{1}{2^{l}}\left(\begin{array}{l}
l-1 \\
\left\lfloor\frac{l-1}{2}\right\rfloor
\end{array}\right)\right),
$$

but, they do not prove that the bound is sharp. The goal of this paper is to provide a new proof of this result, showing that the bound is indeed sharp and that the signed graphs that meet this bound are unique (up to switching).

\section{Preliminaries}

A signed graph, $\Sigma$, is a pair $(G, \sigma)$ where $G$ is a graph, and $\sigma: E(G) \rightarrow\{+,-\}$. We call $\sigma$ the sign function of $\Sigma$. The graph $G$ is called the underlying graph of $\Sigma$, and is written $|\Sigma|$. A signed graph $(G, \sigma)$ is called bipartite if its underlying graph is bipartite. In this paper the bipartition of the vertices is chosen in advance and will be denoted by the pair $(L, R)$. An edge is positive if $\sigma(e)=+$, and negative otherwise. The set of negative edges of $\Sigma$ is denoted $E^{-}(\Sigma)$, and the set of positive edges is $E^{+}(\Sigma)$. The set $E_{v}^{-}$is the set of negative edges incident to vertex $v$, and $E_{v}^{+}$is the set of positive edges incident to $v$. Similarly, we partition the neighbors of $v, N_{v}$, into $N_{v}^{+}$and $N_{v}^{-}$. If $N_{v}=N_{v}^{+}, v$ is called all positive. If every vertex of $(G, \sigma)$ is all positive, then the signed graph is the all positive signed graph $+G$. A circle in $\Sigma$ is positive if the product of its edge labels is positive. A signed graph $\Sigma$ is said to be balanced if every circle in $\Sigma$ is positive.

If $U \subseteq V(\Sigma)$, the graph obtained by negating the edges in the cut $\left[U, U^{c}\right]$ is a switching of $\Sigma$ and is denoted $\Sigma^{U}$. A signed graph $\Sigma^{\prime}$ is switching equivalent to $\Sigma$ if $\Sigma^{\prime}=\Sigma^{U}$ for some $U \subseteq V$. The set of signed graphs switching equivalent to $\Sigma$ is called the switching class of $\Sigma$, written $[\Sigma]$. It is well known that the switching class $[\Sigma]$ contains $+G$ if and only if $\Sigma=(G, \sigma)$ is balanced.

The frustration index of a signed graph $\Sigma, f(\Sigma)$, is the minimum number of edges whose deletion yields a balanced signed graph. If $\Sigma$ is balanced then $f(\Sigma)=0$. It is easy to show that

$$
f(\Sigma)=\min _{\Sigma^{\prime} \in[\Sigma]}\left|E^{-}\left(\Sigma^{\prime}\right)\right|
$$

For more information on signed graphs, one can refer to [11].

\section{Graph Cuts and Frustration Index}

Let us consider an arbitrary signed graph $\Sigma$ and a cut $\left[U, U^{c}\right]$ of the underlying graph of $\Sigma$. The weight of $\left[U, U^{c}\right]$, written $\omega\left[U, U^{c}\right]$, is equal to the number of positive edges in $\left[U, U^{c}\right]$ minus the number of negative edges in $\left[U, U^{c}\right]$. A cut is said to be negative if $\omega\left[U, U^{c}\right]$ is negative. 
Lemma 1. Let $U \subseteq V$. The number of negative edges in $\Sigma^{U}$ is $\left|E^{-}(\Sigma)\right|+\omega\left[U, U^{c}\right]$.

Proof. The edges of $\left[U, U^{c}\right]$ are the only edges of $\Sigma$ that change signs when switching $U$. Therefore the number of negative edges of $\Sigma^{U}$ is equal to the number of negative edges of $\Sigma$ not in $\left[U, U^{c}\right]$ plus the number of positive edges of $\Sigma$ in $\left[U, U^{c}\right]$. This is the same as the number of negative edges of $\Sigma$ minus the number of negative edges in $\left[U, U^{c}\right]$ plus the number of positive edges in $\left[U, U^{c}\right]$. Therefore the number of negative edges of $\Sigma^{U}$ is equal to the number of negative edges of $\Sigma$ plus $\omega\left[U, U^{c}\right]$.

Theorem 2. The frustration index of $\Sigma$ is $\left|E^{-}(\Sigma)\right|+\min _{U \subseteq V} \omega\left[U, U^{c}\right]$.

By equation (1), the frustration index of $\Sigma$ is the minimum over $U \subseteq V$ of the number of negative edges of $\Sigma^{U}$. By Lemma 1, we know that the number of negative edges in $\Sigma^{U}$ is equal to the number of negative edges in $\Sigma$ plus $\omega\left[U, U^{c}\right.$. Thus to minimize the number of negative edges in $\Sigma^{U}$ we must minimize $\omega\left[U, U^{c}\right]$.

It is important to note that computing the minimum weight of a cut is NP-hard when you allow negative weights, so using this theorem to find frustration index is not much of an improvement over checking all $2^{|V|}$ switchings.

Definition 3. A signed graph $\Sigma$ is said to be reduced if no switching of $\Sigma$ has fewer negative edges.

Note that when $\Sigma$ is reduced, its frustration index is equal to the number of negative edges of $\Sigma$.

Lemma 4. A signed graph $\Sigma$ is reduced if and only if $\omega\left[U, U^{c}\right]$ is non-negative for each $U \subseteq V(\Sigma)$.

Proof. If there is a $U \subseteq V$ such that $\omega\left[U, U^{c}\right]$ is negative, then $\Sigma^{U}$ has fewer negative edges, and $\Sigma$ is not reduced. Alternatively if $\omega\left[U, U^{c}\right]$ is always non-negative, then every switching of $\Sigma$ has at least as many negative edges, and $\Sigma$ is reduced.

Definition 5. For a graph $G$, the maximum frustration of $G$, denoted $F_{\max }(G)$, is the maximum value of $f(G, \sigma)$ over all signings $\sigma$ of $G$.

In order to determine the value of $F_{\max }(G)$ for a graph $G$, we need only look at those signings of $G$ that are reduced.

From here on we consider an arbitrary signed bipartite graph $\Sigma$.

Definition 6. Let $G=(L, R, E)$ be a bipartite graph and let $S \subseteq L$. An $S$-cut is a cut of $G$ of the form $\left[S \cup T,(S \cup T)^{c}\right]$ where $T \subseteq R$.

Lemma 7. Let $\Sigma=(L, R, E, \sigma)$ be a signed bipartite graph. For each $S \subseteq L$, the set

$$
T:=\left\{v \in R|| S \cap N_{v}^{+}|-| S \cap N_{v}^{-}|\leqslant| S^{c} \cap N_{v}^{+}|-| S^{c} \cap N_{v}^{-} \mid\right\}
$$

minimizes the weight of an S-cut. The weight of this minimum $S$-cut is given by

$$
w(S)=\sum_{v \in R} \min \left(\left|S \cap N_{v}^{+}\right|-\left|S \cap N_{v}^{-}\right|,\left|S^{c} \cap N_{v}^{+}\right|-\left|S^{c} \cap N_{v}^{-}\right|\right) .
$$


Proof. Let $v \in R$ be a vertex with type $\left(N_{v}^{+}, N_{v}^{-}\right)$. For any $T \subseteq R$, if $v \in T$, then $E_{v}$ contributes $\left|S \cap N_{v}^{+}\right|-\left|S \cap N_{v}^{-}\right|$to the weight of the cut. Otherwise $E_{v}$ contributes $\left|S^{c} \cap N_{v}^{+}\right|-\left|S^{c} \cap N_{v}^{-}\right|$to the weight of the cut. To minimize the weight of the $S$-cut, $T$ must be a set of vertices $v$ such that for each $v \in T,\left|S \cap N_{v}^{+}\right|-\left|S \cap N_{v}^{-}\right| \leqslant\left|S^{c} \cap N_{v}^{+}\right|-\left|S^{c} \cap N_{v}^{-}\right|$.

Since replacing $S$ by $S^{c}$ in equation (2) does not change the sum, $w(S)$ is equal to $w\left(S^{c}\right)$.

Let $(P, N)$ be a pair of disjoint subsets of $L$ and let $\mathfrak{T}(L)$ be the collection of all such pairs. If we define

$$
\mathbf{w}_{S}(P, N):=\min \left(|S \cap P|-|S \cap N|,\left|S^{c} \cap P\right|-\left|S^{c} \cap N\right|\right),
$$

then we only need to count the number of right vertices such that $(P, N)=\left(N_{v}^{+}, N_{v}^{-}\right)$in order to compute $w(S)$. If $\mathbf{x}_{\Sigma}(P, N)$ is the number of such vertices, then we can rewrite $w(S)$ as follows:

$$
w(S)=\sum_{(P, N)} \mathbf{x}_{\Sigma}(P, N) \mathbf{w}_{S}(P, N)=\mathbf{x}_{\Sigma} \cdot \mathbf{w}_{S} .
$$

Observe that if $S \subseteq L$, then $\mathbf{w}_{S}(P, N)=\mathbf{w}_{S \cap(P \cup N)}(P, N)$ because the formula for $\mathbf{w}_{S}(P, N)$ depends only on the intersection of $S \cap P$ and $S \cap N$.

Remark 8. If we are given a positive integer vector $\mathbf{x} \in \mathbb{R}^{\mathfrak{T}(L)}$, we can construct a signed graph so that $\mathbf{x}_{\Sigma}=\mathbf{x}$. Start with $\Sigma=((L, \varnothing, \varnothing), \sigma)$. For each $(P, M)$ such that $\mathbf{x}(P, M)$ is non-zero, add $\mathbf{x}(P, M)$ new vertices to $R$ and new edges signed so that $N_{v}^{+}=P$ and $N_{v}^{-}=M$. Now we have a signed $K_{l, r}$ and $\mathbf{x}_{\Sigma}=\mathbf{x}$. Such a signed bipartite graph is called the corresponding signed graph of $\mathbf{x}$.

Theorem 9. Let $\Sigma$ be a signed bipartite graph with underlying bipartite graph $(L, R, E)$. Then $\Sigma$ is reduced if and only if $\mathbf{w}_{S} \cdot \mathbf{x}_{\Sigma}$ is non-negative for all $S \subseteq L$.

Proof. The value of $w(S)$ is the minimum over all $T \subseteq R$ of $\omega\left[S \cup T,(S \cup T)^{c}\right]$. Let $T$ be any set that minimizes the value of $w(S)$. If we switch the cut $\left[S \cup T,(S \cup T)^{c}\right]$, the size of $E^{-}$will increase by $w(S)$. Therefore $\left|E^{-}\left(\Sigma^{S \cup T}\right)\right|=\left|E^{-}(\Sigma)\right|+w(S)$. If $\Sigma$ is reduced, every switching of $\Sigma$ will have more negative edges. Thus $w(S) \geqslant 0$ for all $S$. Since $\mathbf{w}_{S} \cdot \mathbf{x}_{\Sigma}=w(S), \Sigma$ is reduced if and only if $\mathbf{w}_{S} \cdot \mathbf{x}_{\Sigma}$ is non-negative for all $S \subseteq L$.

Lemma 10. Let $S \subseteq P \cup N$ for some type $(P, N)$. Then

$$
w_{S}(P, N)= \begin{cases}|S \Delta N|-|N| & \text { if }|S \Delta N| \leqslant \frac{1}{2}|P \cup N|, \\ \left|S^{c} \Delta N\right|-|N| & \text { if }|S \Delta N| \geqslant \frac{1}{2}|P \cup N| .\end{cases}
$$

Proof. This is just separating the two possibilities from equation (3) and replacing $|S|-$ $2|S \cap N|$ by $|S \Delta N|-|N|$.

Lemma 11. Let $S \subseteq P \cup N$ for some type $(P, N)$ such that $|N| \leqslant|P|$. Then

$$
w_{S}(P, N)= \begin{cases}-w_{S}(P \Delta S, N \Delta S) & \text { if }|S \Delta N| \leqslant \frac{1}{2}|P \cup N|, \\ -w_{S}(N \Delta S, P \Delta S) & \text { if }|S \Delta N| \geqslant \frac{1}{2}|P \cup N| .\end{cases}
$$


Proof. If $|N| \leqslant|P|$ then $|N| \leqslant \frac{1}{2}|P \cup N|$. By equation 4,

$$
w_{S}(P \Delta S, N \Delta S)=|N|-|S \Delta N|=-w_{S}(P, N) .
$$

If $|N| \geqslant|P|$, then $|P| \geqslant \frac{1}{2}|P \cup N|$. By equation 4,

$$
w_{S}(N \Delta S, P \Delta S)=|P|-|S \Delta P|=|P|-\left|S^{c} \Delta N\right|=-w_{S}(P, N) .
$$

Let $L$ be a finite set. The set $\mathfrak{B}(L):=\left\{\left\{S, S^{c}\right\} \mid S \subseteq L\right\}$ is the set of bipartitions of $L$. If $\left\{S, S^{c}\right\}$ and $\left\{T, T^{c}\right\}$ are two elements of $\mathfrak{B}(L)$, then the symmetric difference $\left\{S, S^{c}\right\} \Delta\left\{T, T^{c}\right\}$ is defined to be $\left\{S \Delta T, S^{c} \Delta T\right\}$. Note that the symmetric difference of two bipartitions is commutative so $\mathfrak{B}(L)$ is an abelian group.

Theorem 12. For each bipartition $\{P, N\} \in \mathfrak{B}(L)$, let $(P, N)$ be ordered so that $|P| \geqslant$ $|N|$. Then for any $S \subseteq L$ the following holds:

$$
\sum_{\{P, N\} \in \mathfrak{B}(L)} \mathbf{w}_{S}(P, N)=0 .
$$

Proof. By equation (5), if $|S \Delta N| \leqslant \frac{1}{2}|P \cup N|$, then $\mathbf{w}_{S}(P, N)$ and $\mathbf{w}_{S}(P \Delta S, N \Delta S)$ cancel. Otherwise, $|S \Delta N|>\frac{1}{2}|P \cup N|$ and $\mathbf{w}_{S}(P, N)$ will cancel with $\mathbf{w}_{S}(N \Delta S, P \Delta S)$. Since only one of the latter two can appear in the sum, the sum is zero.

\section{A Cone of Reduced Configurations}

For a complete bipartite graph $K_{l, r}=(L, R, E)$ and signing $\sigma$, each vertex $v \in R$ satisfies $N_{v}^{+} \cup N_{v}^{-}=L$, so we need only consider the pairs in $\mathfrak{B}(L)$. Since $\mathbf{w}_{S}(P, N)=\mathbf{w}_{S^{c}}(P, N)$, we only need to check one cut for each bipartition $\left\{S, S^{c}\right\}$ of $L$ to determine if a signed complete bipartite graph is reduced. Let $\mathcal{K}_{l}$ be the $\mathfrak{B}(L) \times \mathfrak{B}(L)$ matrix whose entry in the $\left(\left\{S, S^{c}\right\},\{P, N\}\right)$ position is the weight contributed by a vertex of type $(P, N)$ to a minimum $S$-cut. That is $\left(\mathcal{K}_{L}\right)_{\left\{S, S^{c}\right\},\{P, N\}}=\mathbf{w}_{S}(P, N)$.

Theorem 13. Let $\Sigma$ be a signed complete bipartite graph with left vertex set $L$. Then $\mathcal{K}_{L} \cdot \mathbf{x}_{\Sigma} \geqslant \mathbf{0}$ if and only if $\Sigma$ is reduced.

Proof. By Theorem 9, $\Sigma$ is reduced if and only if $\mathbf{w}_{S} \cdot \mathbf{x}_{\Sigma} \geqslant 0$ for all $S \subseteq L$.

Let $l=|L|$, define $\mathscr{P}_{l}$ to be the set of vectors in the first orthant of $\mathbb{R}^{\mathfrak{B}(L)}$ satisfying $\mathcal{K}_{L} \cdot \mathbf{x} \geqslant 0$. Also, define $\mathbf{f} \in \mathbb{R}^{\mathfrak{B}(L)}$ as the vector such that

$$
\mathbf{f}(P, N):=\min (|P|,|N|)
$$

In order translate frustration index of a bipartite graph to a geometric setting, we need the following definition.

Definition 14. The frustration of a vector $\mathbf{x} \in \mathscr{P}_{l}$ is $\mathbf{f} \cdot \mathbf{x}$. 
For an integer vector $\mathrm{x}$ in $\mathscr{P}_{l}$, the frustration of $\mathrm{x}$ is the frustration index of the corresponding signed graph. Let $\mathscr{P}_{l, r}$ be the set of vectors of $\mathbf{x} \in \mathscr{P}_{l}$ such that $\mathbf{x} \cdot \mathbf{1}=$ $r$. Here the integer vectors correspond to reduced signings of $K_{l, r}$. We are looking to maximize $\mathbf{f} \cdot \mathbf{x}$ subject to $\mathcal{K}_{L} \cdot \mathbf{x} \geqslant \mathbf{0}$ and $\mathbf{x} \cdot \mathbf{1}=r$ where $\mathbf{x}$ is a non-negative integer vector. That is, we have the integer linear program:

$$
\max \left\{\mathbf{f} \cdot \mathbf{x} \mid \mathbf{x} \geqslant \mathbf{0}, \mathcal{K}_{L} \cdot \mathbf{x} \geqslant \mathbf{0}, \mathbf{x} \in \mathbb{Z}^{\mathfrak{B}(L)}\right\} .
$$

Let $\tilde{\mathcal{K}}_{L}$ be the matrix obtained from $\mathcal{K}_{L}$ by replacing the zero row with the all ones vector. Since the only row of $\mathcal{K}_{L}$ that is all zero is the row corresponding to $\varnothing$, this is well defined.

Lemma 15. If the matrix $\tilde{\mathcal{K}}_{L}$ is invertible, then $\frac{r}{2^{l-1}} \mathbf{1}$ is the unique vertex that maximizes $\mathbf{f} \cdot \mathbf{x}$ in $\mathscr{P}_{l, r}$.

Proof. If $\tilde{\mathcal{K}}_{L}$ is invertible, then $\frac{r}{2^{l-1}} \mathbf{1}$ is the only vertex of $\mathscr{P}_{l, r}$ that satisfies $\tilde{\mathcal{K}}_{L} \mathbf{x}=r \mathbf{e}_{\varnothing}$. The columns of $\tilde{\mathcal{K}}_{L}^{-1}$ give the directions in which we can travel in $\mathscr{P}_{l, r}$ by violating a single hyperplane equality. This means $\tilde{\mathcal{K}}_{l}^{-1} \cdot \mathbf{f}$ gives the change in frustration from traveling in each of these directions away from $v$. Define $\mathbf{b} \in \mathbb{R}^{\mathfrak{B}(L)}$ by letting $\mathbf{b}(\varnothing, L)=2^{l-1}-1$ and $\mathbf{b}\left(S, S^{c}\right)=-1$ otherwise. Using equation (5) we can see that $\tilde{\mathcal{K}}_{l} \cdot \mathbf{b}=2^{l-1} \mathbf{f}$, and thus $\tilde{\mathcal{K}}_{l}^{-1} \cdot \mathbf{f}=\frac{1}{2^{l-1}} \mathbf{b}$. This means that traveling along any edge of $P_{l, r}$ incident to the point $\frac{r}{2^{l-1}} \mathbf{1}$ will reduce the frustration of the resulting vector. Since $\frac{r}{2^{l-1}} \mathbf{1}$ is the only vertex satisfying

$$
\tilde{\mathcal{K}}_{L} \mathbf{X}= \begin{cases}r & \text { if }\left\{S, S^{c}\right\}=\{\varnothing, L\} \\ 0 & \text { otherwise }\end{cases}
$$

it must maximize $\mathbf{f} \cdot \mathbf{x}$ in $\mathscr{P}_{l, r}$.

Let $\mathcal{S}_{L}$ be the $\mathfrak{B}(L) \times \mathfrak{B}(L)$ matrix such that

$$
\left(\mathcal{S}_{L}\right)_{\left\{S, S^{c}\right\},\{P, M\}}=\min \left(|S \Delta M|,\left|S^{c} \Delta M\right|\right) .
$$

Note that $\mathcal{S}_{L}=\mathcal{K}_{L}+\mathbf{1}^{T} \mathbf{f}$. This is because the $\left(\left\{S, S^{c}\right\},\{P, M\}\right)$ entry of $\mathcal{K}_{L}$ is

$$
\min \left(|S \Delta M|-|M|,\left|S^{c} \Delta M\right|-|M|\right),
$$

where $|P| \leqslant|M|$. Thus the first row of $\mathcal{S}_{L}$ is $\mathbf{f}$.

Lemma 16. If $\mathcal{S}_{L}$ is invertible, then the null space of $\mathcal{K}_{L}$ is spanned by $\mathbf{1}$.

Proof. Let $\mathbf{y}$ be a non-zero element in the null space of $\mathcal{S}_{L}$. By equation (4), we know that $\mathcal{S}_{L}-\mathbf{1}^{T} \mathbf{f}=\mathcal{K}_{L}$. We also know that $\mathbf{f}$ is the first row of $\mathcal{S}_{L}$, which means $\mathbf{f} \cdot \mathbf{y}=0$. Therefore, $\mathbf{y}$ is in the null space of $\mathcal{K}_{L}$. If $\mathcal{S}_{L}$ is invertible, then the only way to be in the null space of $\mathcal{K}_{L}$ is to be a solution of $\mathcal{S}_{L}=c \mathbf{1}$ for some constant $c$. If $\mathcal{S}_{L}$ is invertible, then there can only be one solution to this equation. This proves that $\mathcal{K}_{L}$ has nullity 1 .

Definition 17. Let $\mathfrak{G}$ be a group, and let $\mathcal{M}$ be a $\mathfrak{G} \times \mathfrak{G}$ matrix. $\mathcal{M}$ is said to be $\mathfrak{G}$-invariant if there is a class function $f: \mathfrak{G} \rightarrow \mathbb{C}$ such that $\mathcal{M}_{g, h}=f\left(h g^{-1}\right)$. 
Since $\left(\mathcal{S}_{L}\right)_{\left\{S, S^{c}\right\},\{P, N\}}=\mathbf{f}\left(\left\{S, S^{c}\right\} \Delta\{P, N\}\right), \mathcal{S}_{L}$ is $\mathfrak{B}(L)$-invariant.

Let $\chi$ be a character of $\mathfrak{G}$. Define $\lambda_{\chi}$ by

$$
\lambda_{\chi}:=\sum_{g \in \mathfrak{G}} \chi(g) f(g)
$$

Theorem 18 (Eigenvalues of Invariant Matrices [2]). If $\mathcal{M}$ is a $\mathfrak{G}$-invariant matrix, then the eigenvectors of $\mathcal{M}$ are the characters of $\mathfrak{G}$. Furthermore, if $\chi$ is a character of $\mathfrak{G}$, then $\lambda_{\chi}$ is the eigenvalue of $\chi$.

Definition 19. Let $0 \leqslant i \leqslant l$. Define

$$
\lambda_{i}(l):=\sum_{T \subseteq[l-1]}(-1)^{|T \cap[i]|} \min (|T|, l-|T|) .
$$

Theorem 20. Let $0 \leqslant i \leqslant l$. Then $\lambda_{i}(l)$ is an eigenvalue of $\mathcal{S}_{L}$. Furthermore, the multiplicity of $\lambda_{i}(l)$ is $\left(\begin{array}{c}l-1 \\ i\end{array}\right)$.

Proof. Since $\mathfrak{B}(L)$ is isomorphic to the power set of $[l-1], \chi_{S}(T)=(-1)^{|T \cap S|}$ is a character of $\mathfrak{B}(L)$ for each $S \subseteq[l-1]$. By equation (13),

$$
\lambda_{S}(l)=\sum_{T \subseteq[l-1]}(-1)^{|T \cap S|} \min (|T|, l-|t|) .
$$

Furthermore, the value of $\lambda_{S}(l)$ depends only on the size of $S$. Therefore,

$$
\lambda_{S}(l)=\sum_{T \subseteq[l-1]}(-1)^{|T \cap\{1,2, \ldots,|S|\}|} \min (|T|, l-|t|) .
$$

Since $\lambda_{S}(l)=\lambda_{i}(l), \lambda_{i}(l)$ is an eigenvalue of $\mathcal{S}_{L}$. To prove that the multiplicity of $\lambda_{i}(l)$ is $\left(\begin{array}{c}l-1 \\ i\end{array}\right)$, note that there are $\left(\begin{array}{c}l-1 \\ i\end{array}\right)$ subsets of $[l-1]$ of size $i$.

Lemma 21. If $l$ is odd and $0<i<l$, then $\lambda_{i}(l)=\frac{1}{2} \lambda_{i}(l+1)$.

Proof. Let us compute the value of $\lambda_{i}(l+1)$ by breaking the summation into two parts. The first part is over those subsets such that $l \notin T$, and the second part is over subsets such that $l \in T$.

$$
\begin{aligned}
& \lambda_{i}(l+1)=\sum_{T \subseteq[l-1]}(-1)^{|T \cap[i]|} \min (|T|, l+1-|T|) \\
& +\sum_{T \subseteq[l-1]}(-1)^{|(T \cup\{l\}) \cap[i]|} \min (|T \cup\{l\}|, l+1-|T \cup\{l\}|) .
\end{aligned}
$$

Since $i<l,(-1)^{|(T \cup\{l\}) \cap[i]|}=(-1)^{|T \cap[i]|}$. So except for the +1 that appears on different sides to the two minimums, each term looks like the corresponding term in $\lambda_{i}(l)$. We will move the +1 from the minimum in the first term into a new sum when $l+1-|T|<|T|$. 
Similarly, we will move the +1 from the minimum in the first term into a new sum when $l+1-|T| \geqslant|T|$. Thus

$$
\lambda_{i}(l+1)=2 \lambda_{i}(l)+\sum_{\substack{T \subseteq[l-1] \\ l<2|T|}}(-1)^{|T \cap[i]|}+\sum_{\substack{T \subseteq[l-1] \\ l>2|T|}}(-1)^{|T \cap[i]|} .
$$

Since $l$ is odd, the two remaining pieces cancel, and we are done.

Lemma 22. If $i$ is even and $0<i \leqslant l$, then $\lambda_{i}(l)=\lambda_{i-1}(l)$.

Proof. We will show that $\lambda_{i}(l)-\lambda_{i-1}(l)=0$ when $i$ is even. Note that

$$
\lambda_{i}(l)-\lambda_{i-1}(l)=\sum_{T \subseteq[l-1]} \min (|T|, l-|T|)\left((-1)^{|T \cap[i]|}-(-1)^{|T \cap[i-1]|}\right) .
$$

If $i \notin T$, then $\left((-1)^{|T \cap[i]|}-(-1)^{|T \cap[i-1]|}\right)$ is zero. If $i \in T$, then this difference is equal to $-2(-1)^{|T \cap[i-1]|}$. Thus the only non-zero terms left in the sum are when $i \in T$. Therefore,

$$
\lambda_{i}(l)-\lambda_{i-1}(l)=-2 \sum_{\substack{T \subseteq[l-1] \\ i \in T}} \min (|T|, l-|T|)(-1)^{|T \cap[i-1]|} .
$$

Because $i-1$ is odd, the parities of $T \cap[i]$ and $([l-1] \backslash T) \cap[i-1]$ are different. Also, $\min (|T|, l-|T|)=\min (|([l-1] \backslash T) \cup\{i\}|, l-|([l-1] \backslash T) \cup\{i\}|)$. Thus the remaining terms in the sum pair up and cancel.

Lemma 23. If $0<i \leqslant l$ is odd and $l=2 k$, then

$$
\lambda_{i}(l)=(-1)^{k} \frac{2^{k-1}}{(k-1) !} \prod_{t=1}^{k}(2(k+t)-i) .
$$

In particular, $\lambda_{i}(l) \neq 0$.

Theorem 24. Let $0<i \leqslant l$, if $i$ is even, then

$$
\lambda_{i}(l)=(-1)^{\lceil l / 2\rceil} \frac{2^{\lfloor l / 2\rfloor-1}}{(\lceil l / 2\rceil-1) !} \prod_{t=1}^{\lceil l / 2\rceil}(2(\lceil l / 2\rceil+t)+(i-1)) .
$$

If $i$ is odd, then

$$
\lambda_{i}(l)=(-1)^{\lceil l / 2\rceil} \frac{2^{\lfloor l / 2\rfloor-1}}{(\lceil l / 2\rceil-1) !} \prod_{t=1}^{\lceil l / 2\rceil}(2(\lceil l / 2\rceil+t)+i) .
$$

Proof. By Lemma 23, the formula is correct when $l$ is even and $0<i \leqslant l$ is odd. By Lemma 21, if $l$ is odd then $\lambda_{i}(l)=\frac{1}{2} \lambda_{i}(l+1)$. By Lemma 22, $\lambda_{i}(l)=\lambda_{i-1}(l)$ when $i$ is even (except for when $i=0$ ). Thus formula (17) is correct. 
Lemma 25. The matrix $\mathcal{S}_{L}$ is invertible.

Proof. By Theorem 24, the eigenvalues are given by equations (16) and (17). Because each factor in each of the products is odd, the eigenvalues are never zero. The only value not accounted for by Theorem 24 is when $i=0$, but equation (14) shows $\lambda_{0}(l)$ is positive. Therefore $\mathcal{S}_{L}$ is invertible.

Theorem 26. The matrix $\tilde{\mathcal{K}}_{L}$ is invertible.

Proof. By lemma 16, the null space of $\mathcal{K}_{L}$ is spanned by $\mathbf{1}$ and the rank of $\tilde{\mathcal{K}}_{L}$ is one greater than the rank of $\mathcal{K}_{L}$. Therefore $\tilde{\mathcal{K}}_{L}$ is invertible.

Theorem 27. Given $l, r \geqslant 0$,

$$
F_{\max }\left(K_{l, r}\right) \leqslant \frac{l r}{2}\left(1-\frac{1}{2^{l-1}}\left(\begin{array}{c}
l-1 \\
\left\lfloor\frac{l-1}{2}\right\rfloor
\end{array}\right)\right),
$$

with equality if and only if $r$ is a positive integer multiple of $2^{l-1}$.

Proof. Combining Lemma 15 and Theorem $26, \frac{r}{2^{l-1}} \mathbf{1}$ is the unique vertex that maximizes $\mathbf{f} \cdot \mathbf{x}$ in $\mathscr{P}_{l, r}$. Therefore, $\mathbf{f} \cdot \frac{r}{2^{l-1}} \mathbf{1}$ is the maximum value of $\mathbf{f} \cdot \mathbf{x}$ over $\mathscr{P}_{l, r}$. Whenever $\frac{r}{2^{l-1}}$ is an integer, we have an integral solution to the linear $\operatorname{program} \max \left\{\mathbf{f} \cdot \mathbf{x} \mid \mathbf{x} \in \mathscr{P}_{l, r}\right\}$. Because non-negative integral vectors in $\mathscr{P}_{l, r}$ correspond to signed complete bipartite graphs, we have a signed $K_{l, r}$ with maximum frustration. If $\frac{r}{2^{l-1}}$ is not an integer, the optimal solution to the linear program is not integer, so the solution to the integer program is strictly smaller than the solution to the linear program.

It remains to be shown that the maximum frustration index of $K_{l, r}$ is bounded by the expression in inequality (18). To do this, we need only compute the value of $\mathbf{f} \cdot \frac{r}{2^{l-1}} \mathbf{1}$. Note that

$$
\mathbf{f} \cdot \mathbf{1}=\sum_{\{P, N\} \in \mathfrak{B}(L)} \min (|P|,|N|) .
$$

By convention, $|N| \leqslant|P|$ so we can use binomial coefficients to write the sum over the size of $N$. If $l$ is even, we add up the sizes of each set of size less than $l / 2$ and half the sets of size $l / 2$.

$$
\begin{aligned}
\mathbf{f} \cdot \mathbf{1} & =\sum_{i=0}^{\frac{1}{2} l}\left(\begin{array}{c}
l \\
i
\end{array}\right) i-\frac{1}{2}\left(\begin{array}{c}
l \\
\frac{1}{2} l
\end{array}\right) \\
& =\sum_{i=0}^{\left\lfloor\frac{l-1}{2}\right\rfloor}\left(\begin{array}{c}
l \\
i+1
\end{array}\right)(i+1)-\frac{1}{2}\left(\begin{array}{c}
l \\
\frac{1}{2} l
\end{array}\right) \frac{1}{2} l .
\end{aligned}
$$


Now replace $\left(\begin{array}{c}l \\ i+1\end{array}\right)(i+1)$ by $l\left(\begin{array}{c}l-1 \\ i\end{array}\right)$ and $\left(\begin{array}{c}l \\ l / 2\end{array}\right)(l / 2)$ by $l\left(\begin{array}{c}l-1 \\ \left\lfloor\frac{l-1}{2}\right\rfloor\end{array}\right)$ :

$$
\begin{aligned}
\mathbf{f} \cdot \mathbf{1} & =l\left(\sum_{i=0}^{\left\lfloor\frac{l-1}{2}\right\rfloor}\left(\begin{array}{c}
l-1 \\
i
\end{array}\right)-\frac{1}{2}\left(\begin{array}{l}
l-1 \\
\left\lfloor\frac{l-1}{2}\right\rfloor
\end{array}\right)\right) \\
& =l\left(2^{l-2}-\frac{1}{2}\left(\begin{array}{c}
l-1 \\
\left\lfloor\frac{l-1}{2}\right\rfloor
\end{array}\right)\right) .
\end{aligned}
$$

If $l-1$ is odd, we have no sets of size $\frac{1}{2} l$ so instead

$$
\mathbf{f} \cdot \mathbf{1}=\sum_{i=0}^{\left\lfloor\frac{1}{2} l\right\rfloor}\left(\begin{array}{l}
l \\
i
\end{array}\right) i=\sum_{i=0}^{\frac{l-1}{2}}\left(\begin{array}{l}
l \\
i
\end{array}\right) i
$$

Now replace $\left(\begin{array}{l}l \\ i\end{array}\right) i$ by $l\left(\begin{array}{c}l-1 \\ i-1\end{array}\right)$, so

$$
\mathbf{f} \cdot \mathbf{1}=l \sum_{i=0}^{\frac{l-1}{2}-1}\left(\begin{array}{c}
l-1 \\
i
\end{array}\right)=l\left(2^{l-2}-\frac{1}{2}\left(\begin{array}{c}
l-1 \\
\left\lfloor\frac{l-1}{2}\right\rfloor
\end{array}\right)\right) .
$$

Multiplying equations (22) and (24) by $\frac{r}{2^{l-1}}$ gives the desired bound.

Perhaps the most interesting thing about this theorem is that the bound is a linear function of $r$. Since the bound is achieved periodically (with period $2^{l-1}$ ), it is also the best linear upper bound.

Using Stirling's formula, you can show that the maximum frustration of a signed $K_{l, r}$ is bounded above by

$$
\frac{\operatorname{lr}}{2}\left(1-\sqrt{\frac{2}{\pi l}}\right)
$$

Corollary 28. The maximum frustration of $K_{n, n}$ is bounded above by

$$
\frac{n^{2}}{2}\left(1-\frac{1}{2^{n-1}}\left(\begin{array}{c}
n-1 \\
\left\lfloor\frac{n-1}{2}\right\rfloor
\end{array}\right)\right)
$$

Proof. Let $l=r=n$ in Theorem 27.

Note that this bound is a strict upper bound, even if (26) is an integer. This is because the only way the bound of Theorem 27 can be obtained is when $r=2^{l-1}$.

\section{Maximum Frustration of Complete Bipartite Graphs}

Let $\mathscr{C}_{\tilde{\mathcal{K}}_{L}}$ be the set of points in $\mathbb{R}^{\mathfrak{B}(L)}$ that satisfy $\tilde{\mathcal{K}}_{L} \mathbf{x} \geqslant 0$.

Theorem 29. Let $r$ be a positive integer, and let $\mathbf{x}_{0} \in \mathscr{C}_{\tilde{\mathcal{K}}_{L}}$ be an integer point that maximizes the value of $\mathbf{f} \cdot \mathbf{x}$ over all $\mathbf{x} \in \mathscr{C}_{\tilde{\mathcal{K}}_{L}}$ such that $\mathbf{x} \cdot \mathbf{1}=r$. Then $\mathbf{x}_{0}+n \mathbf{1}$ is an integer point that maximizes $\mathbf{f} \cdot \mathbf{x}$ over all $\mathbf{x} \in \mathscr{C}_{\tilde{\mathcal{K}}_{L}}$ such that $\mathbf{x} \cdot \mathbf{1}=r+n 2^{l-1}$. 
Proof. Since $\tilde{\mathcal{K}}_{L}$ is invertible, the cone $\mathscr{C}_{\tilde{\mathcal{K}}_{L}}$ must be simple (and simplicial). This is because the only way to get a generating ray is by taking the intersection of all but one of the defining hyperplanes. Because $\mathscr{C}_{\tilde{\mathcal{K}}_{L}}$ is simple, every integer point in the cone is equal to an integer linear combination of generating vectors plus some integer point in the fundamental parallelepiped. Thus the columns of $\tilde{\mathcal{K}}_{L}^{-1}$ are the generating vectors of the cone $\mathscr{C}_{\tilde{\mathcal{K}}_{L}}$. By Lemma 15 , each generating vector except the first has negative frustration. Thus, maximum integer solutions must be integer multiples of 1 plus an integer point from inside the fundamental domain.

Definition 30. Let $\varepsilon_{l, r}$ be the difference between the bound in Theorem 27 and $F_{\max }\left(K_{l, r}\right)$.

By Theorem 27, for any positive integer $k$ there is a signed graphs that attain the bound with equality when $r=k 2^{l-1}$. Therefore, $\varepsilon_{l, k 2^{l-1}}$ is zero for every positive integer $k$.

Theorem 31. The maximum frustration of $K_{5, r}$ is equal to $\left\lfloor\frac{25}{16} r\right\rfloor-\varepsilon_{r}$, where

$$
\varepsilon_{r}= \begin{cases}1 & \text { if } r \equiv 2,4,9,13 \quad(\bmod 16) \\ 0 & \text { otherwise }\end{cases}
$$

except for $r=1,3$, where $\varepsilon_{5,1}=\varepsilon_{5,3}=1$.

Proof. By Theorem 29, if we have a set of solutions to

$$
\max \left\{\mathbf{f} \cdot \mathbf{x} \mid \mathbf{x} \in \mathscr{C}_{\tilde{\mathcal{K}}_{[5]}} \cap \mathbb{Z}^{\mathfrak{B}([5])}, \mathbf{x} \cdot \mathbf{1}=r\right\}
$$

for $0 \leqslant r \leqslant 16$, adding $k \mathbf{1}$ to any of these solutions will give a solution to

$$
\max \left\{\mathbf{f} \cdot \mathbf{x} \mid \mathbf{x} \in \mathscr{C}_{\tilde{\mathcal{K}}_{[5]}} \cap \mathbb{Z}^{\mathfrak{B}([5])}, \mathbf{x} \cdot \mathbf{1}=16 k+r\right\} .
$$

So long as one of the solutions $\mathbf{x}_{\max }$ is positive, we have a signed graph with frustration index $\mathbf{f} \cdot \mathbf{x}_{\max }$. Using GLPK, [7], we can construct a list of integer solutions to (28) when $0 \leqslant r \leqslant 16$. Since the least entry of any of these solutions is -1 , adding $k \mathbf{1}$ with $k \geqslant 1$ will give a non-negative integer optimum solution. The only $r$ for which there are no non-negative optima to the integer program of (28) are 1 and 3.

In [10], Solé and Zaslavsky computed $F_{\max }\left(K_{l, r}\right)$ when $l \leqslant 5$. My method provides another proof of their results. It also corrects their formula for $F_{\max }\left(K_{5, r}\right)$ when $r=15$. In [10], they say $F_{\max }\left(K_{5,15}\right)=22$, when in fact there is a signed $K_{5,15}$ which achieves the bound of 23 from Theorem 27.

Example 32. By finding a solution to the integer programming problem

$$
\max \left\{\mathbf{f} \cdot \mathbf{x} \mid \mathbf{x} \in \mathscr{C}_{\tilde{\mathcal{K}}_{[5]}}, \mathbf{x} \cdot \mathbf{1}=15\right\}
$$


one can construct a reduced signed $K_{5,15}$ whose frustration index is 23 . Its bipartite adjacency matrix is

$$
\left(\begin{array}{ccccccccccccccc}
1 & -1 & 1 & 1 & 1 & 1 & -1 & -1 & -1 & -1 & 1 & 1 & 1 & 1 & 1 \\
1 & 1 & -1 & 1 & 1 & 1 & -1 & 1 & 1 & 1 & -1 & -1 & -1 & 1 & 1 \\
1 & 1 & 1 & -1 & 1 & 1 & 1 & -1 & 1 & 1 & -1 & 1 & 1 & -1 & -1 \\
1 & 1 & 1 & 1 & -1 & 1 & 1 & 1 & -1 & 1 & 1 & -1 & 1 & -1 & 1 \\
1 & 1 & 1 & 1 & 1 & -1 & 1 & 1 & 1 & -1 & 1 & 1 & -1 & 1 & -1
\end{array}\right)
$$

The frustration index is the number of negative entries since the graph is reduced. One can check that the corresponding signed graph is reduced by checking that the weights of the 16 minimum $S$-cuts are all non-negative. The weights of these cuts are all either 0,1 , or 2 .

Theorem 33. The maximum frustration of $K_{6, r}$ is equal to $\left\lfloor\frac{66}{32} r\right\rfloor-\varepsilon_{6, r}$, where

$$
\varepsilon_{6, r}= \begin{cases}0 & \text { if } r \equiv 6,12,14 \quad(\bmod 16) \\ 0 & \text { if } r \equiv 0,8,26,31 \quad(\bmod 32), \\ 1 & \text { if } r \equiv 5 \quad(\bmod 8), \\ 1 & \text { if } r \equiv 2,4,7,9,11 \quad(\bmod 16), \\ 1 & \text { if } r \equiv 10,15,16,24 \quad(\bmod 32), \\ 2 & \text { if } r \equiv 1,3,17,19 \quad(\bmod 32)\end{cases}
$$

The only exception is when $r=6$, where $\varepsilon_{6,6}=1$.

Theorem 34. The maximum frustration of $K_{7, r}$ is equal to $\left\lfloor\frac{154}{64} r\right\rfloor-\varepsilon_{r}$, where

$$
\varepsilon_{r}=\left\{\begin{array}{cc}
0 & \text { if } r \equiv 7,12,14,16,17,22 \\
& 24,26,27 \quad(\bmod 32) \\
0 \quad \text { if } r \equiv & 3,8,18,34,36,38 \\
& 43,51,63 \quad(\bmod 64)
\end{array}\right.
$$

With exceptions when $r \in\{3,14,17,18,36,49\}$ or $r \in\{1,2,5,10,26\}$, where $\varepsilon_{r}$ is 1 in the first case, and 2 in the second.

While the methods of integer programming are capable of producing results for $l \leqslant 7$ quickly, computational time increases exponentially, so in general this is not a great method for arriving at new formulas. Hopefully further examination of the cone $\mathscr{C}_{\tilde{\mathcal{K}}_{L}}$ will yield better results. In particular, it would be nice to show that $\varepsilon_{l, r}$ is bounded above by a linear term in $l$. 


\section{References}

[1] R. Abelson and M. Rosenberg, Symbolic psycho-logic: a model of attitudinal cognition. Behavioral Sci, 3 (1958) 1-13.

[2] P. Diaconis, Group Representations in Probability and Statistics. Institute of Mathematical Statistics, 1988.

[3] J. Carlson and D. Stolarski, The correct solution to Berlekamp's switching game, Discrete Math., Vol. 287 (2004).

[4] P. C. Fishburn and N. J. A. Sloane, The solution to Berlekamp's switching game, Discrete Math., 74 (1989), 263-290..

[5] R. Graham and N. J. A. Sloane, On the covering radius of codes. IEEE Trans. Inform. Theory, IT-31 (1985) 385-401.

[6] F. Harary, On the notion of balance of a signed graph. Michigan Math. Journal, 2 (1953-54) 143-146.

[7] A. Makhorin, GNU Linear Programming Kit 4.33. GNU Software Foundation, http: //www.gnu.org/software/glpk/glpk.html

[8] M. Petersdorf, Einige bemerkungen über vollstängidge bigraphen. Wiss. Z. Techn. Hochsch. Ilmenau, 12 (1966) 257-260.

[9] N. J. A. Sloane and S. Plouffe, The Encyclopedia of Integer Sequences, Academic Press, 1995

[10] P. Solé and T. Zaslavsky, A coding approach to signed graphs. SIAM J. Discrete Math. 4 (1994) 544-553.

[11] T. Zaslavsky, Signed graphs. Discrete Appl. Math. 4 (1982) 47-74. 